\title{
TRATAMENTO DA EXTROFIA DE BEXIGA: OSTEOTOMIA POSTERIOR DOS OSSOS ILÍACOS E FECHAMENTO DA PELVE COM CINTA DE NÁILON
}

\author{
TREATMENT OF BLADDER EXSTROPHY: POSTERIOR OSTEOTOMY OF ILIAC BONES AND \\ CLOSING OF THE PELVIS WITH A NYLON BRACES
}

Francisco Carlos Salles Nogueira', Bernardo Luiz Fornaciari Ramos², Leonardo Pelucci Machado ${ }^{3}$, Manoel Firmato de Almeida ${ }^{4}$, Moacir Astolfo Tibúrcio ${ }^{5}$, Luiz Fernando Silveira Parrela ${ }^{6}$, Tiago Kfuri Maciel ${ }^{7}$, Fellipe Ferreira Valle ${ }^{8}$

\section{RESUMO}

Objetivo: Avaliou-se o resultado obtido em nove correções cirúrgicas de extrofia de bexiga em pacientes pediátricos. Métodos: Foi utilizada osteotomia posterior dos ossos ilíacos seguida pelo fechamento da pelve com cinta de náilon. Os procedimentos foram realizados em dois tempos cirúrgicos distintos com $48 \mathrm{~h}$ de intervalo. Resultado: A média de idade foi de 17,6 meses. Cinco pacientes eram do sexo masculino. O seguimento médio foi de 32 meses. Conclusão: Os resultados finais foram animadores, mesmo nos pacientes que apresentaram complicações pós-operatórias como infecções e deiscência.

Descritores - Extrofia Vesical; Osteotomia; Pelve; Resultado de Tratamento

\section{ABSTRACT}

Objective: The authors evaluated the results obtained in nine surgical correction of bladder exstrophy in pediatric patients. Methods: We used posterior osteotomy of pelvic iliac bones followed by closing with a nylon brace. The procedures were performed in two different surgical times with intervals of 48 hours. Results: The mean age was 17.6 months. Five patients were male. The mean follow-up was 32 months. Conclusion: The final results were encouraging even in patients who had postoperative complications such as infection and dehiscence.

Keywords - Bladder Exstrophy; Osteotomy; Pelvis; Treatment Outcome

\section{INTRODUÇÃO}

A extrofia de bexiga é uma anomalia congênita rara decorrente de falha da fusão dos tecidos da linha média da pelve durante a embriogênese e caracteriza-se por má formação da região inferior da parede abdominal envolvendo o trato geniturinário e o sistema musculoesquelético ${ }^{(1,2)}$. Apresenta incidência estimada em 1:30.000 a 1:50.000 nascidos vivos, sendo duas a três vezes mais frequente no sexo masculino ${ }^{(3-5)}$. Na extrofia vesical clássica a parede anterior da parte posterior da bexiga está exposta e alterações como epispádia, displasia dos músculos do assoalho pélvico, pênis curto ou clitóris bifurcado acompanham o quadro clinico ${ }^{(1,4)}$. O dismorfismo da pelve representado pela diástase da sínfise púbica, expansão lateral dos ossos inominados e rotação lateral dos acetábulos está invariavelmente presente nessa afecção ${ }^{(6)}$.

1 - Ortopedista e Traumatologista SBOT; Professor FCM-MG; Mestre em Cirurgia; Chefe do Serviço de Ortopedia Pediátrica da Santa Casa de Belo Horizonte, MG, Brasil.

2 - Médico Graduado pela Universidade Federal de Minas Gerais (UFMG); Ortopedista e Traumatologista SBOT; Médico Assistente do Serviço de Ortopedia Pediátrica da Santa Casa de Belo Horizonte, MG, Brasil.

3 - Ortopedista e Traumatologista SBOT; Médico Assistente do Serviço de Ortopedia Pediátrica da Santa Casa de Belo Horizonte, MG, Brasil.

4 - Médico Chefe do Serviço de Cirurgia Infantil da Santa Casa de Belo Horizonte, MG, Brasil.

5 - Médico Assistente do Serviço de Cirurgia Infantil da Santa Casa de Belo Horizonte, MG, Brasil.

6 - Médico Residente do Terceiro Ano em Ortopedia e Traumatologia da Santa Casa de Belo Horizonte, MG, Brasil.

7 - Médico Residente do Terceiro Ano em Ortopedia e Traumatologia da Santa Casa de Belo Horizonte, MG, Brasil.

8 - Médico Residente do Terceiro Ano em Ortopedia e Traumatologia da Santa Casa de Belo Horizonte, MG, Brasil..

Trabalho realizado no Departamento de Ortopedia Pediátrica da Santa Casa de Belo Horizonte, MG, Brasil.

Correspondência: Bernardo Luiz Fornaciari Ramos, Rua Deputado Álvaro Sales, 95, Santo Antonio - 30350-250 - Belo Horizonte, MG, Brasil. E-mail: bernardolframos@ hotmail.com

Trabalho recebido para publicação: 24/08/2011, aceito para publicação: 31/08/2011.

Os autores declaram inexistência de conflito de interesses na realização deste trabalho / The authors declare that there was no conflict of interest in conducting this work 
Em virtude da maioria das estruturas urológicas estarem presentes ou serem bífidas, é possível a reconstrução ${ }^{(1,7)}$. Entretanto, a menos que a sínfise púbica seja aproximada, a reconstrução urológica estará sujeita a maior incidência de complicações, como formação de fistulas ou recidivas. Essas parecem ser causadas pela tensão exercida sobre os tecidos moles durante o fechamento cirúrgico, que pode ser aliviada com reparo da sínfise $^{(1,4,8)}$. Em recém-nascidos com menos de 72 horas de vida e diástase púbica inferior a quatro centímetros, pode ser possível aproximação da sínfise. Caso contrário, é recomendada osteotomia pélvica ${ }^{(5,9)}$.

Neste trabalho objetivou-se avaliar os resultados de nove procedimentos para tratamento da extrofia de bexiga utilizando osteotomia posterior dos ossos ilíacos seguida por fechamento da pelve com cinta de náilon.

\section{MATERIAIS E MÉTODO}

No período de março de 2005 a julho de 2010 realizaramse nove cirurgias para o tratamento da extrofia de bexiga na Santa Casa de Belo Horizonte. Os dados dos pacientes foram coletados exclusivamente dos prontuários médicos. Observou-se a idade, o sexo, o exame físico e os exames radiográficos, como exemplificados na Figura 1. A tomografia computadorizada foi utilizada apenas em situações específicas para melhor definição da morfologia pélvica.

A técnica cirúrgica aplicada foi constante e a equipe cirúrgica composta por ortopedistas e cirurgiões pediátricos. Em um primeiro tempo, com o paciente sob anestesia geral e em decúbito ventral, realizou-se a osteotomia posterior dos ossos ilíacos tipo O'Phelan, dois e meio centímetros lateralmente à articulação sacroilíaca ${ }^{(1)}$. Após

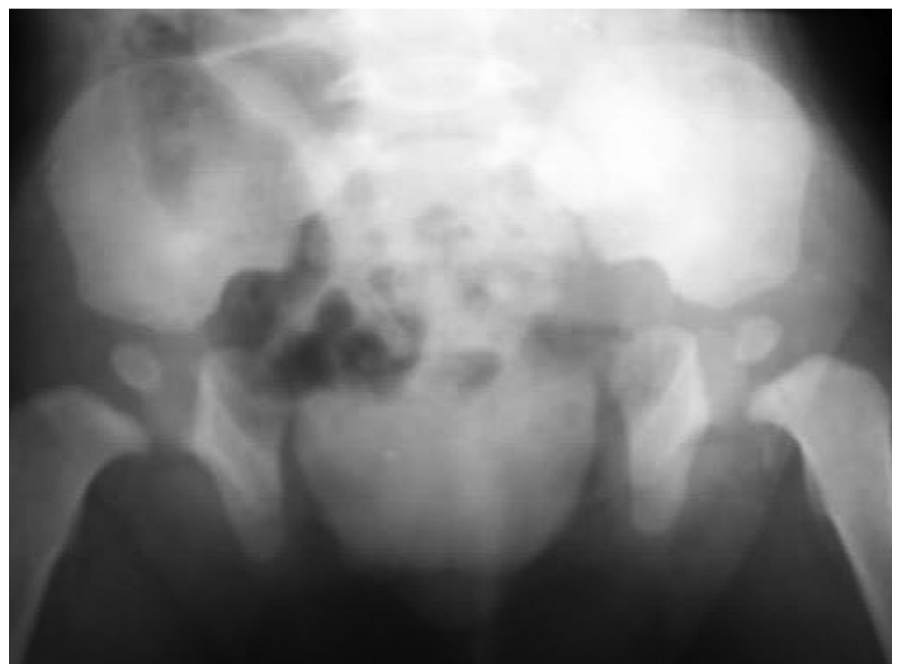

Figura 1 - Radiografia da bacia de um dos pacientes operados. Incidência AP pré-operatória. 48h realizou-se a reconstrução das estruturas anteriores da parede abdominal, trato urinário e aproximação da sínfise púbica. Essa aproximação foi efetuada através de uma cinta de náilon passada pelos forames obturatórios. À medida em que foi realizado o tensionamento da cinta, a pelve se fechava progressivamente.

O material de síntese utilizado é composto por poliamida (náilon), que apresenta as seguintes propriedades: alta resistência mecânica, baixo custo, boa resistência à fadiga, ao desgaste e à abrasão, além de baixo coeficiente de atrito. É considerado um material biologicamente inerte. A cinta apresenta dentições que impedem o deslocamento em sentido inverso ao de sua progressão e, na medida em que se realiza sua tração pela ponta lisa, ocorre diminuição do diâmetro do material e consequente aproximação entre os ossos púbicos (Figura 2).

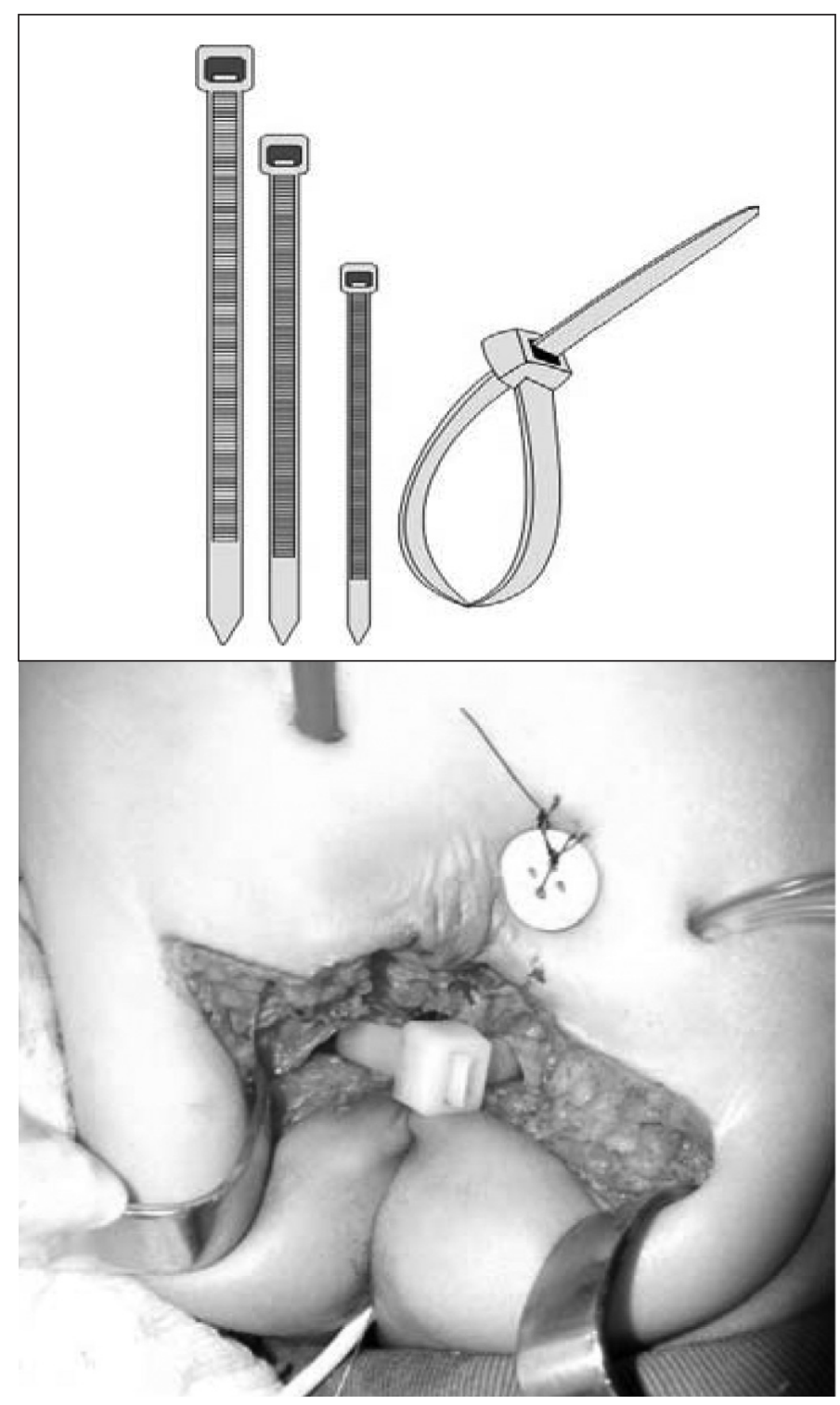

Figura 2 - Desenho da cinta de náilon e seu aspecto cirúrgico final. 
$\mathrm{Na}$ análise estatística foram consideradas a distribuição de frequência, as medidas de tendência central e as comparações entre proporções. O projeto de pesquisa foi aprovado pelos órgãos competentes das instituições envolvidas. Em relação aos aspectos éticos, seguiram- se as normas do Ministério de Saúde para pesquisa em crianças e adolescentes e as dos comitês de ética das instituições. A utilização do consentimento pós-informado foi dispensável, por se tratar de análise envolvendo apenas o exame de anotações médicas contidas em documentos hospitalares, que manteve, assegurado em sua metodologia, o direito ao sigilo.

\section{RESULTADOS}

Foram avaliados nove prontuários de pacientes submetidos a tratamento cirúrgico para correção da extrofia de bexiga no período entre março de 2005 e julho de 2010. Utilizou-se a técnica descrita previamente em todos os casos. A média de idade foi de 17,6 meses (quatro a 60 meses). Cinco pacientes eram do sexo masculino $(55,6 \%)$ e quatro, do feminino $(44,4 \%)$. O seguimento médio foi de 32 meses (10 a 60 meses). Em oito pacientes $(88,9 \%)$ foi possível uma boa aproximação da sínfise púbica. Três pacientes precisaram ser submetidos à retirada do material de síntese antes do previsto devido infecção da ferida operatória e deiscência de sutura. Nestes casos foram utilizados gessos em abdução e rotação interna para manutenção do fechamento da pelve, previamente obtido sem prejuízo do resultado final. Em um paciente não foi obtido sucesso no fechamento da pelve com o método descrito, havendo necessidade da realização de osteotomia anterior do osso ilíaco. Ao se tentar a aproximação da sínfise púbica com a cinta de náilon, houve fratura de um dos ramos púbicos superiores e o procedimento não foi concretizado. Apesar disso, o paciente apresentou boa evolução. Os resultados foram confirmados através de radiografias da bacia. Duas pacientes, gêmeas siamesas, apresentavam pelve com displasia importante e evoluíram com alteração rotacional da pelve, manifestada por rotação externa dos membros inferiores. A Tabela 1 expõe os parâmetros avaliados, os resultados conseguidos e as complicações pós-operatórias. As Figuras 3 e 4 demonstram alguns dos resultados ectoscópicos e radiográficos conseguidos.
Tabela 1 - Resultados do tratamento cirúrgico de nove crianças com extrofia de bexiga.

\begin{tabular}{c|c|c|c|c|c}
\hline Paciente & Idade & Sexo & $\begin{array}{c}\text { Tempo de } \\
\text { seguimento }\end{array}$ & Resultado & Complicação \\
\hline 1 & $20 \mathrm{~m}$ & $\mathrm{M}$ & $60 \mathrm{~m}$ & $\begin{array}{c}\text { Boa } \\
\text { aproximação* }\end{array}$ & $\begin{array}{c}\text { Deiscência e } \\
\text { infecção }\end{array}$ \\
\hline 3 & $18 \mathrm{~m}$ & $\mathrm{~F}$ & $50 \mathrm{~m}$ & $\begin{array}{c}\text { Boa } \\
\text { aproximação }\end{array}$ & Não houve \\
\hline 4 & $10 \mathrm{~m}$ & $\mathrm{~F}$ & $20 \mathrm{~m}$ & $\begin{array}{c}\text { Boa } \\
\text { aproximação }\end{array}$ & Não houve \\
\hline 5 & $12 \mathrm{~m}$ & $\mathrm{M}$ & $16 \mathrm{~m}$ & $\begin{array}{c}\text { Boa } \\
\text { aproximação* houve }\end{array}$ & $\begin{array}{c}\text { Deiscência e } \\
\text { infecção }\end{array}$ \\
\hline 6 & $9 \mathrm{~m}$ & $\mathrm{M}$ & $15 \mathrm{~m}$ & $\begin{array}{c}\text { Realizada } \\
\text { osteotomia/sem } \\
\text { aproximação } \\
\text { com a cinta }\end{array}$ & Fratura ramo \\
púbico \\
\hline 7 & $8 \mathrm{~m}$ & $\mathrm{M}$ & $13 \mathrm{~m}$ & $\begin{array}{c}\text { Boa } \\
\text { aproximação }\end{array}$ & Não houve \\
\hline 8 & $4 \mathrm{~m}$ & $\mathrm{~F}$ & $8 \mathrm{~m}$ & $\begin{array}{c}\text { Boa } \\
\text { aproximação }\end{array}$ & Não houve \\
\hline 9 & $60 \mathrm{~m}$ & $\mathrm{M}$ & $7 \mathrm{~m}$ & $\begin{array}{c}\text { Boa } \\
\text { aproximação* }\end{array}$ & Deiscência e \\
infecção
\end{tabular}

*Nesses pacientes foi conseguida boa aproximação da pelve com a cinta de náilon. O material foi retirado devido infecção e deiscência. No entanto, o fechamento pélvico obtido foi mantido no gesso.

\section{DISCUSSÃO}

A osteotomia posterior dos ossos ilíacos descrita por O'Phelan ${ }^{(10)}$ e utilizada no tratamento da extrofia de bexiga é uma técnica de grande popularidade e consagrada pela literatura quanto aos resultados proporcionados ${ }^{(5,11)}$ tendo sido realizada em todos os nove casos descritos. Diversos tipos de osteotomia foram desenvolvidos para ajudar na aproximação da sínfise púbica e diminuir a tensão da parede abdominal durante seu fechamento.

Os primeiros estudos foram realizados por O'Phelan ${ }^{(10)}$, que descreveu os resultados de osteotomias posteriores dos ossos ilíacos seguidas de aproximação da sínfise púbica. Tratava-se de uma série de 25 pacientes com seguimento de cinco anos. Ele relatou que o procedimento reduziu as taxas de deiscência e melhorou os resultados da reconstrução geniturinária. A principal complicação observada foi a separação tardia da sínfise. Tal complicação não foi observada no presente estudo. Mas esse fato poderia ser explicado pelo curto seguimento médio. Deiscência foi uma complicação em três casos $(33,3 \%)$. Lesões do nervo ciático ou da artéria glútea não foram descritas pelo autor em questão, o que coincide com os achados dos noves casos operados.

Aadalen et $a l^{(12)}$ revisaram uma série de 100 pacien- 


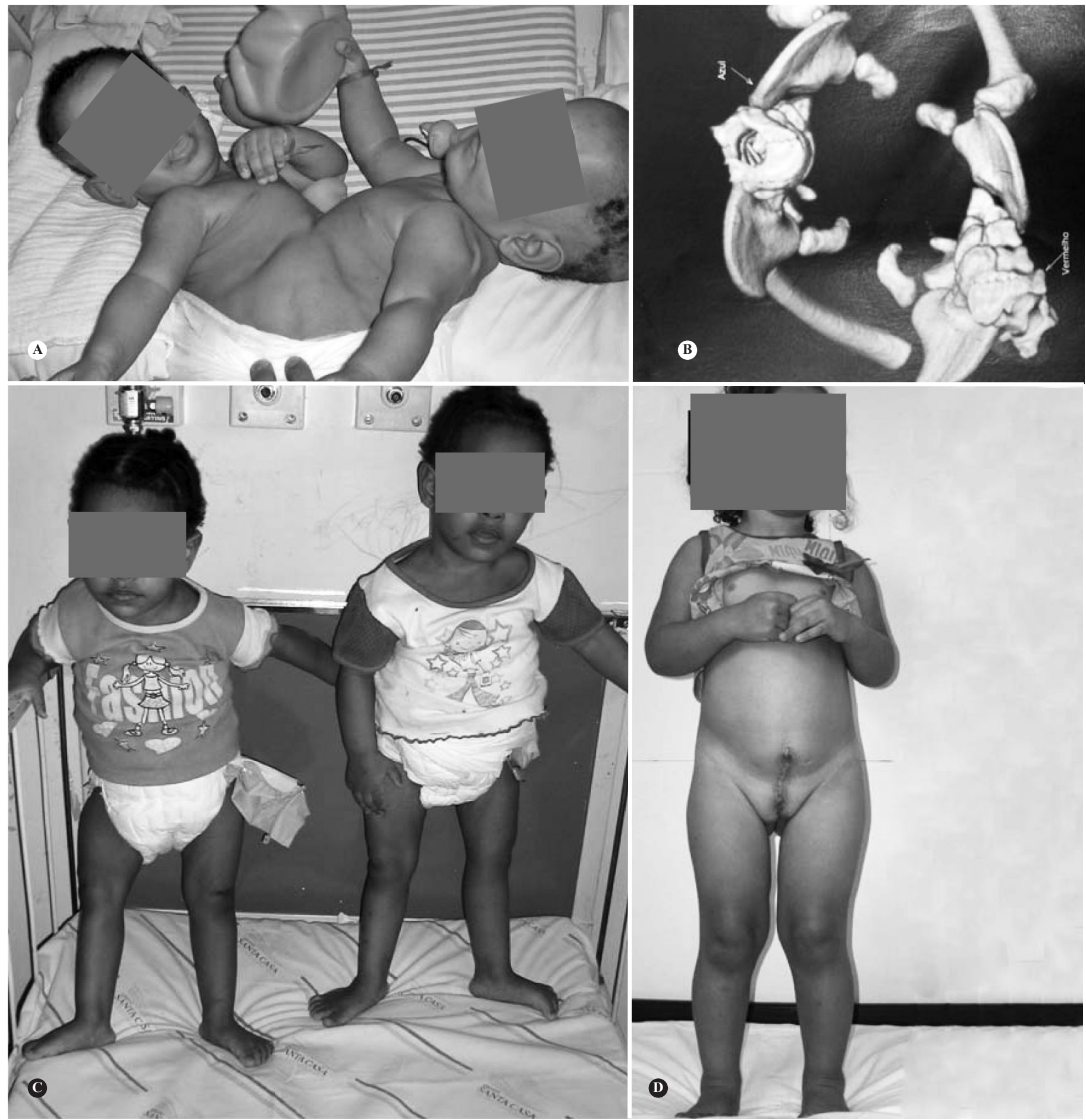

Figura 3 - A) Gêmeas siamesas - casos 2 e 3 da Tabela 1. B) Reconstrução tomográfica das pelves de ambas. C) Aspecto final após a separação total delas e do tratamento da extrofia de bexiga. D) Ectoscopia de uma outra criança operada.

tes submetidos à osteotomia tipo O'Phelan com seguimento de cinco a 18 anos e relataram $45 \%$ de excelentes resultados na aproximação da sínfise púbica e seis casos de piora gradual da simetria pélvica. Foi observado, também, que os pacientes com diastase inferior a dois centímetros tiveram melhores resultados quanto à continência urinaria ${ }^{(13)}$. Observou-se índice similar de excelentes resultados (55\%), levando-se em conta, exclusivamente, pacientes que não apresentaram qualquer tipo de complicação pós-operatória. Mas, se avaliarmos exclusivamente o fechamento pélvico, esse resultado seria ainda superior com oito pacientes $(88,9 \%)$ atingindo totalmente o objetivo inicial. A continência urinária não foi avaliada. 


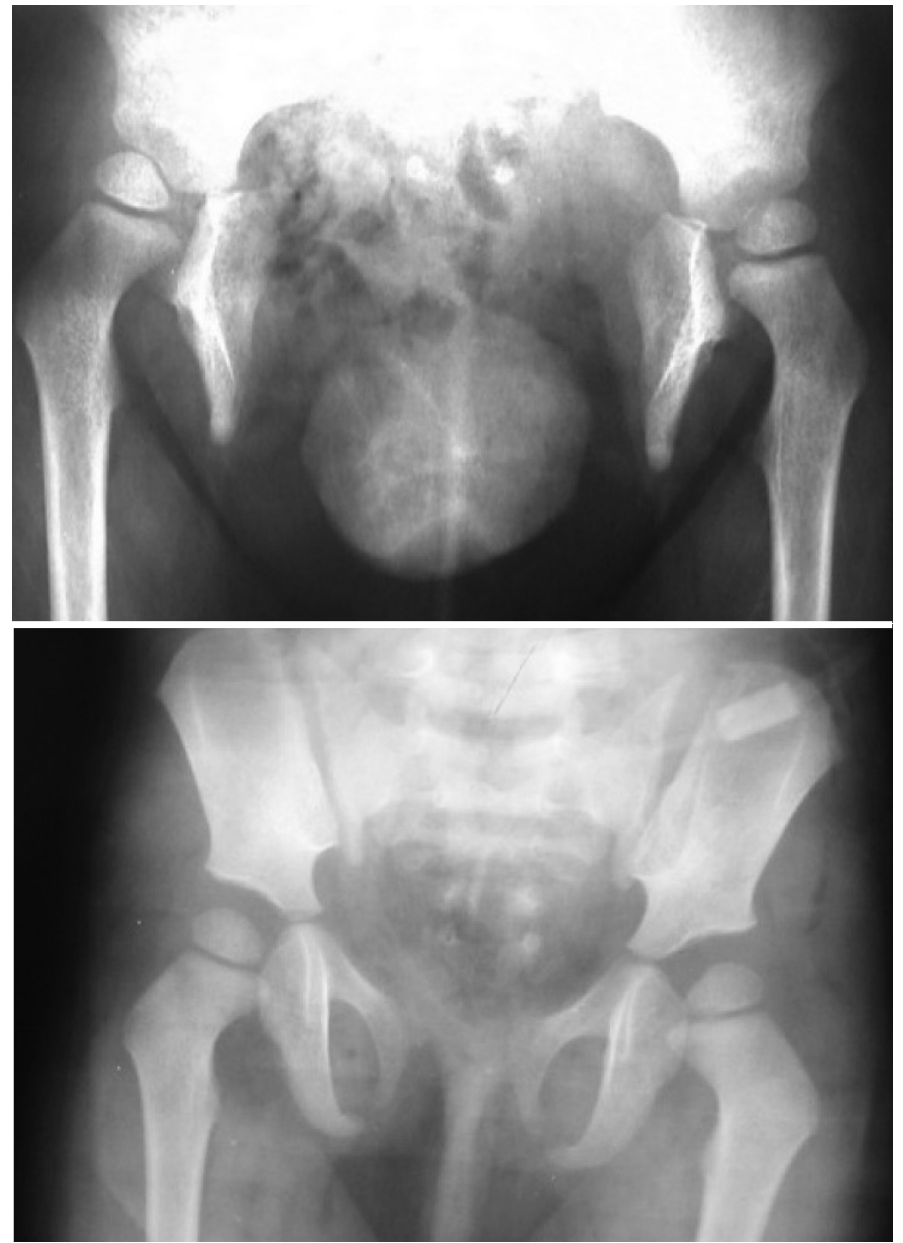

Figura 4 - Radiografia pré-operatória e um mês após o procedimento do caso mostrado acima na Figura 3, D.

Sponseller et $a^{(14)}$ relataram os benefícios da osteotomia na falha da reconstrução sem esse procedimento. Esses autores utilizaram uma osteotomia transversa dos ilíacos semelhante à de Salter, com bons resultados ${ }^{(13,14)}$. Em nenhum caso foi utilizada técnica parecida.

Chiari et $a l^{(11)}$ e Frey e Cohen ${ }^{(15)}$ descreveram uma

\section{REFERÊNCIAS}

1. Beaty $\mathrm{JH}$. Congenital and developmental anomalies of hip and pelvis In: Canale TS. Campbell's operative orthopaedics. 10th ed. St Louis: Mosby; 2003. p. 1118-9.

2. Ramírez E, Calderón M. Osteotomía ilíaca en extrofia vesical. Rev Mex Ortop Traumatol. 1997;11(5):293-6.

3. Segev E, Ezra E, Binyamini Y, Wientroub S, Ben-Chaim J. A combined vertical and horizontal pelvic osteotomy approach for repair of bladder exstrophy: the Dana experience. Isr Med Assoc J. 2004;6(12):749-52.

4. Frey P. Bilateral anterior pubic osteotomy in bladder exstrophy closure. J Urol. 1996;156(2 Pt 2):812-5.

5. Satsuma S, Kobayashi D, Yoshiya S, Kurosaka M. Comparison of posterior and anterior pelvic osteotomy for bladder exstrophy complex. J Pediatr Orthop B. 2006;15(2):141-6.

6. Gökçora IH, Yazar T. Bilateral transverse iliac osteotomy in the correction of neonatal bladder extrophies. Int Surg. 1989;74(2):123-5.

7. Fong Aldama F, Álvarez Núñez R, Santana Valera R, Toledo Martínez E, García Sosa N. Extrofia vesical: difícil y complicado tratamiento en el campo de la Urología y Ortopedia. Rev Medica Electron. 2007;29(2). Disponível em: URL:http://www.cpimtz.sld.cu/revista\%20medica/año\%202007/vol2\%20 2007/tema06.htm nova técnica que consistia na osteotomia pélvica anterior dos ramos púbicos superiores, mostrando ser segura e um bom método alternativo para se obter a aproximação da sínfise sem tensão. McKenna et $a l^{(9)}$ também apresentaram uma nova técnica com osteotomia anterior diagonal dos ilíacos mostrando bons resultados. Um caso necessitou da realização de uma osteotomia anterior para fechamento pélvico, haja vista que houve insucesso na utilização da cinta de náilon. Apresentou boa evolução.

Diferentes métodos de fixação da sínfise púbica após a realização da osteotomia são descritos na literatura. Durante vários anos, o fio de aço e o fixador externo da pelve foram os materiais comumente utilizados para esse fim, porém com índice de complicações significativo. O fio de aço apresenta como principal complicação a secção dos ossos púbicos imaturos. Já o fixador externo tem como complicações a infecção no trajeto $\operatorname{dos}_{\text {pinos }}{ }^{(13)}$, desconforto local, difícil aceitação pelos familiares e necessidade de segundo procedimento para retirada do material. Por outro lado, com a utilização da cinta de náilon não foram observadas complicações associadas ao material e a necessidade de um segundo procedimento se deu apenas nos casos em que ocorreu infecção e deiscência com exposição da mesma.

\section{CONCLUSÃO}

A aproximação da pelve com a cinta de náilon apresenta menor índice de complicações quando comparada com as técnicas que utilizam fixador externo e fio de aço. Até o presente momento mostra resultados animadores, mas necessita de um seguimento maior para confirmação de sua eficácia e identificação de possíveis complicações tardias ainda não identificadas.

8. Giron AM, Arap S, Goes GM. Experiência com osteotomia pélvica na reconstrução cirúrgica da extrofia vesical. Rev Hosp Clin Fac Med Univ Säo Paulo. 1984;39(3):107-12.

9. McKenna PH, Khoury AE, McLorie GA, Churchill BM, Babyn PB, Wedge JH. Iliac osteotomy: a model to compare the options in bladder and cloacal exstrophy reconstruction. J Urol. 1994;151(1):182-6.

10. O'Phalen EH. Iliac Osteotomy in extrophy of the bladder. J Bone Joint Surg Am. 1963;45(7):45:1409-22.

11. Chiari G, Avolio L, Bragheri R. Bilateral anterior pubic osteotomy in bladder exstrophy repair: report of increasing success. Pediatr Surg Int. 2001;17(2-3):160-3.

12. Aadalen RJ, O'Phelan EH, Chisholm TC, McParland FA Jr, Sweetser TH Jr. Exstrophy of the bladder: long-term results of bilateral posterior iliac osteotomies and two-stage anatomic repair, Clin Orthop Relat Res. 1980;(151):193-200.

13. Sponseller PD, Jani MM, Jeffs RD, Gearhart JP. Anterior innominate osteotomy in repair of bladder exstrophy. J Bone Joint Surg Am. 2001;83(2):184-93.

14. Baird AD, Sponseller PD, Gearhart JP. The place of pelvic osteotomy in the modern era of bladder exstrophy reconstruction. J Pediatr Urol. 2005;1(1):31-6.

15. Frey P, Cohen SJ. Anterior pelvic osteotomy. A new operative technique facilitating primary bladder exstrophy closure. $\mathrm{Br} \mathrm{J}$ Urol. 1989;64(6):641-3. 